\title{
Impact of COVID-19 Pandemic on the Life of Indian Doctors: Time for Introspection?
}

\author{
Trupti Nadkarni ${ }^{1}$, Sukhpreet Patel ${ }^{2}$, Amol Pawar ${ }^{3, *}$, Minnie Bodhanwalla ${ }^{4}$
}

Trupti Nadkarni ${ }^{1}$, Sukhpreet Patel ${ }^{2}$, Amol Pawar ${ }^{3, *}$, Minnie Bodhanwalla ${ }^{4}$ ${ }^{1}$ Department of Obstetrics and Gynaecology, Nowrosjee Wadia Maternity Hospital, Parel, Mumbai, Maharashtra, INDIA.

${ }^{2}$ Reproductive Medicine and Research Department, Nowrosjee Wadia Maternity Hospital, Parel, Mumbai, Maharashtra, INDIA.

${ }^{3}$ Nowrosjee Wadia Maternity Hospital, Acharya Donde Marg, Opposite KEM Hospital, Parel, Mumbai, Maharashtra, INDIA.

${ }^{4}$ Chief Executive Officer, Nowrosjee Wadia Maternity Hospital, Parel, Mumbai, Maharashtra, INDIA.

\section{Correspondence}

Dr. Amol Pawar

Department of Obstetrics and Gynaecology, Nowrosjee Wadia Maternity Hospital, Parel, Opposite KEM Hospital, Parel, Mumbai-400012, Maharashtra, INDIA.

Mobile no: +91 9820610319

Email: amolpawar@hotmail.com

History

- Submission Date: 02-01-2021;

- Revised Date: 15-02-2021;

- Accepted Date: 23-03-2021;

DOI : 10.5530/ijmedph.2021.2.15

Article Available online

http://www.jjmedph.org/v11/i2

\section{Copyright}

(C) 2021 Phcog.Net. This is an openaccess article distributed under the terms of the Creative Commons Attribution 4.0 International license.

\begin{abstract}
Introduction: The COVID-19 pandemic is the first of its kind that doctors have encountered in their clinical practice, offering several challenges in all aspects of their lives. The aim of this study was to look into the impact of the COVID-19 pandemic on the personal and professional lives of doctors. Methods: 264 anonymous voluntary responses received within one week of sending 805 requests to Indian doctors in July 2020, through Google forms were included in the study. Results: Of the 264 respondents, $72.3 \%$ continued to stay at home with their families as before, $22 \%$ practiced self-isolation at home and a small proportion of mainly young doctors stayed in a hostel or other accommodation. Majority of the respondents reported an improvement in family life and interpersonal relationships, eating habits, volume of daily exercise and fitness levels. Regarding the financial situation, more than $70 \%$ of doctors were negatively impacted during the pandemic. While a majority of doctors appreciated technological aid in the form of teleconsultation and webinars, most of them questioned their application post COVID-19. Over $40 \%$ of doctors experienced some form of discrimination against them during the pandemic. Conclusion: While healthcare professionals face a unique set of obstacles and dilemmas that require special attention, many have also experienced a silver lining amidst this chaos in the form of family life and lifestyle changes. Regardless, COVID-19 has been a game changer for everyone!.
\end{abstract}

Key words: COVID-19, Healthcare professionals, Doctors, Coronavirus, Lifestyle, Mental Health.

\section{INTRODUCTION}

The Coronavirus pandemic in India is part of the pandemic across the globe caused by the severe acute respiratory syndrome virus 2 (SARS-CoV-2). The first case was reported in India on 30 January 2020. India currently has the largest number of confirmed cases in Asia and the second largest number of confirmed cases in the world after the United States. ${ }^{1}$ On 24 March 2020, a nationwide lockdown was announced, which was then extended to 31 May 2020 in phases. From 1 June 2020, the government started unlocking the country (barring the containment zones) in three phases.

For most practicing doctors, this was their first experience of a pandemic of this magnitude. Shielding themselves while fighting for their patients, updating their knowledge and treatment strategies, adapting their practices and bearing the burden of being a threat to their own families, were just some of the challenges faced by this community. The aim of this study was to analyse some of the impacts of the COVID-19 pandemic on the personal and professional lives of doctors, the frontline workers during this pandemic.

\section{MATERIALS AND METHODS}

We conducted a survey in the last week of July 2020 to analyse the impact of the current COVID-19 pandemic on the life of Indian doctors. The survey was conducted using Google forms and sent via WhatsApp chat messages to 805 Indian doctors in various chat groups. The population surveyed included doctors from different specialities across the country. It was an anonymous survey and confidentiality of the participants was maintained by not including any details of personal identity in the questionnaire like name, email address or telephone number. 264 anonymous participants who voluntarily responded to the survey within one week were included in the study to represent the cross section of doctors across the country. The questionnaire had a total of twelve questions. The first five questions consisted of demographic details like age, gender, years of clinical experience, qualification and area of specialisation. The next three questions focused on details of clinical work done in the pandemic, accommodation and whether discrimination was experienced during the pandemic time as a health care worker. The following two questions focused on areas of impact of the pandemic on personal life and impact on mental health. The participants had to respond in check boxes against various parameters. Participants were also asked about their views on the use of technology in consultation and medical education and whether they felt it held promise for
Cite this article : Nadkarni T, Patel S, Pawar A, Bodhanwalla M. Impact of COVID-19 Pandemic on the Life of Indian Doctors: Time for Introspection?. Int J Med Public Health. 2021;11(2):85-9. 
the future. The last question was an optional one which required the participant to express their views on the changes they would like to bring about in their lives after the pandemic was over. The questions were diversified to reduce response bias and processing bias was reduced by cleaning the data. Some bias in sampling may be prevalent due to respondent effect and neutral responding.

\section{RESULTS}

\section{Demography}

The respondents of the survey were mainly male (64.8\%) with age ranging from 23 to 71 years. Maximum participants were between 40 50 years of age with a median age of 45 years. Of the 264 participants, the majority $(81.4 \%)$ were specialists in different fields, $11.6 \%$ belonged to super speciality fields and $7 \%$ were medical graduates practicing as general practitioners.

A large proportion of respondents (48.9\%) had clinical experience of 10-20 years which signified the peak of their careers. Ninety $(35.6 \%)$ participants were senior doctors with more than 20 years of clinical practice and $15.5 \%$ were doctors who had less than 10 years of clinical experience. While $88.8 \%$ of the respondents continued to practice during the pandemic, the majority of the remaining who refrained from doing so belonged to the high-risk group due to their age.

\section{Impact on home stay}

Of the respondents, the majority $(72.3 \%)$ continued to stay at home with their families as usual.

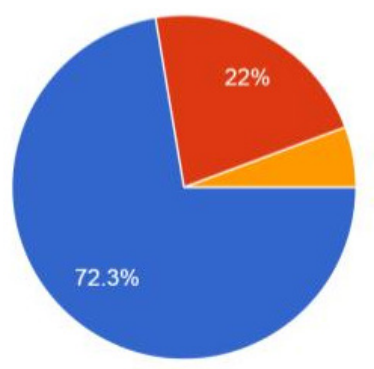

I continued to stay at home as usual I was self isolating at home I was staying in a hostel / other accommodation instead of returning home

Figure 1: Impact of Covid 19 on home stay.
While $22 \%$ practiced self-isolation at home, a small proportion of mainly young doctors (5.7\%) stayed in a hostel or other accommodation (Figure 1). This was probably because of longer hours of duty in COVID-19 wards requiring isolation according to government norms.

\section{Impact on personal life}

Family time: On questioning about the impact of the pandemic on the quality of time spent with family, $64.3 \%$ respondents reported an improvement in family life and interpersonal relationships. On the other hand, $20.8 \%$ and $14.7 \%$ respondents reported worsening and status quo of their family life during the pandemic, respectively.

Eating Habits: $46.2 \%$ respondents reported an improvement in eating habits whereas $18.1 \%$ respondents felt that their eating habits had worsened during the lockdown.

Exercise and fitness: Positive effects on the volume of daily exercise and fitness levels were reported by $47.7 \%$ and $41.2 \%$, respectively. However, $25 \%$ of the doctors reported a decline in their daily exercise and $21.9 \%$ felt there was a deterioration in their physical fitness levels.

Sleep duration and quality: While approximately one-third of the doctors reported an improvement in sleep duration and quality, a similar proportion experienced a deterioration in sleep quality.

The results are summarised in Figure 2.

\section{Impact on monthly expenditure and livelihood}

Regarding income, a vast majority (78.03\%) reported a fall, $18.5 \%$ reported no change and only $3.4 \%$ of the doctors disclosed an increase in their monthly earnings. With respect to monthly expenditure, $48.4 \%$ reported no change as opposed to $23.48 \%$ who reported an increase in expenditure during the pandemic. Considering these proportions, we estimate that the financial situation of more than $70 \%$ of doctors was negatively impacted during the pandemic.

\section{Impact on well-being and mental health}

We inquired into the predominant emotions experienced during the pandemic to give an indication of physician's attitude and well-being in the pandemic. We asked them how often they felt the emotions of fear, anxiety, panic, anger and sadness. While the majority of respondents went through these emotions sometimes during the course of the pandemic,

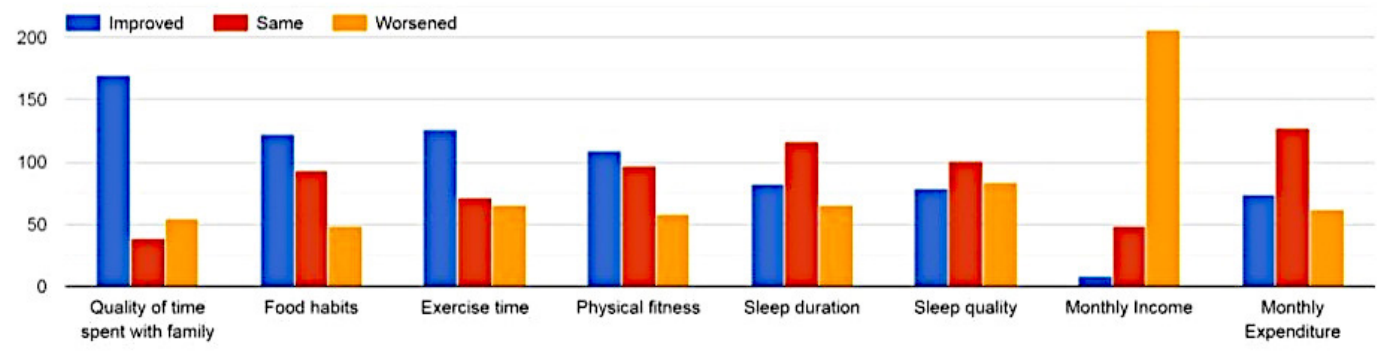

Figure 2: Impact of Covid 19 on personal lives.

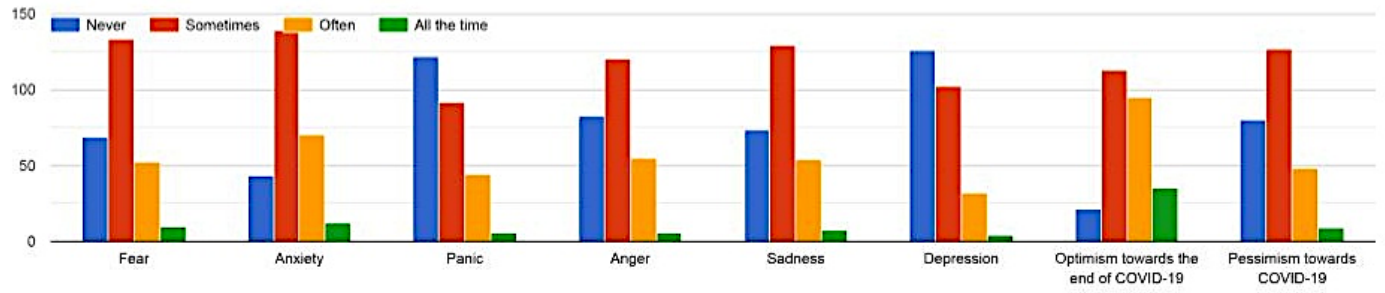

Figure 3: Impact of Covid 19 on mental health. 
we considered the number of respondents going through these emotions often or all the time as representative of their state of mind during the pandemic period. Regarding mental health, 23.04\% respondents felt fear, $31.06 \%$ experienced anxiety, $18.9 \%$ experienced panic, $23.1 \%$ felt anger and $23.2 \%$ experienced sadness often or all the time during the pandemic (Figure 3). This indicates that at least quarter of our study population was experiencing negative mental health during the pandemic.

\section{Impact on professional life}

Teleconsultation: While more than half of the doctors indicated that they would like to continue using teleconsultation post pandemic, $7.9 \%$ voted against its use and one third were unsure whether teleconsultation would continue to have a serious role in consultations in the post pandemic era. At the same time, majority (73.8\%) agreed that teleconsultation cannot replace a face to face consultation with a patient.

Webinars: More than $40 \%$ of the doctors revealed the usefulness of webinars during the pandemic and supported their continuance post pandemic. Nevertheless, one-fifth of the respondents expressed the lack of usefulness of webinars as a tool for medical education and discouraged its continuation after this pandemic (Figure 4).

Discrimination: While more than half (56.8\%) did not feel discriminated against during the pandemic, $35.2 \%$ reported occasional discrimination and only a small proportion (8\%) disclosed discrimination most of the time (Figure 5).

\section{Resolutions for the future}

The last optional question in the survey was optional where participants were asked what changes they would like to make to their life post pandemic. A total of $182(68.9 \%)$ of participants responded. Of the 182 responses to the optional question regarding the changes that the doctors would like to make to their life post pandemic, most of the replies were in favour of making or maintaining the positive changes in their lifestyle. These included better work life balance, improved quality of time with

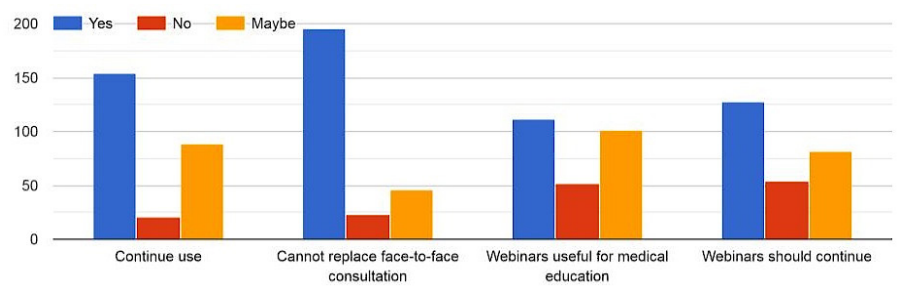

Figure 4: Use of Technology post Covid 19.

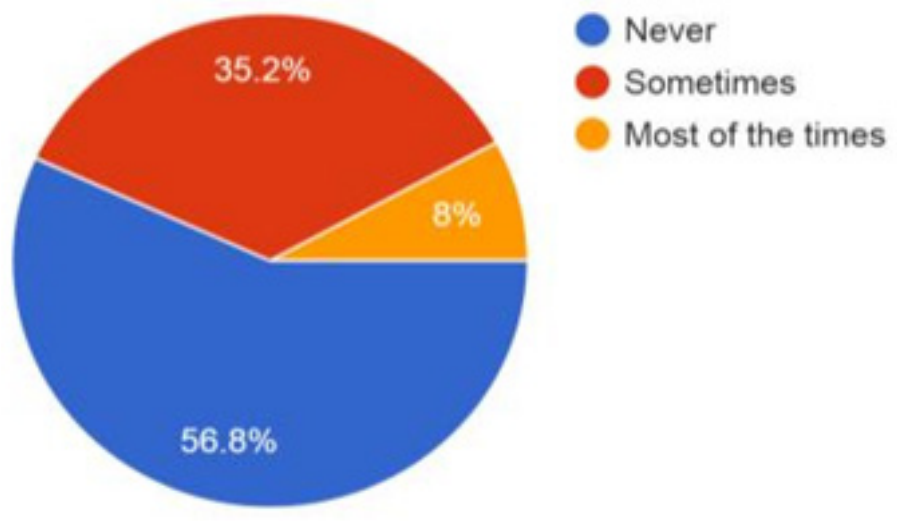

Figure 5: Discrimination during Covid Pandemic. family, focus on health and healthy living, pursuing new hobbies, restricting hours of work and resetting their priorities in life.

\section{DISCUSSION}

\section{Impact on home stay}

One of the foremost worries in the mind of the health care professional during this pandemic is the risk posed to the health of their family members. Self-isolation at home seems to be the ideal which would provide the medical professional the emotional stability of returning to their homes but minimizing risk to family members by maintaining social distancing. However, this is easier said than done and over a prolonged period it is difficult to sustain maintaining the norms of social distancing with family members. In our study, a majority of respondents $72.3 \%$ continued with homestay as usual without social isolation thereby potentially putting these families also at increased risk of exposure. On the other hand, 5.7\% of respondents stayed away from their families and $86.67 \%$ of these reported a worsening of family life. In a review by Nicholas Jones and Catherine Caver to examine whether interventions such as social distancing were effective at reducing the risk of transmitting COVID-19 infection to other household members, they found 87 studies of potential interest focusing on social distancing. They found no studies examining social distancing of asymptomatic health care workers from family members at home and they cautioned health care workers against this step given the increased risk of isolation and anxiety it could create. They advised symptomatic health care workers to self-isolate. In addition, they also advised that healthcare workers could take other precautions to protect their families, such as hand hygiene both at work and at home - and using appropriate personal protective gear at the workplace. ${ }^{2}$

\section{Impact on lifestyle}

Medical professionals have habitually neglected their own health in favour of professional and personal obligations. ${ }^{3}$ The 2007 Physician Health survey conducted by E Frank and C Segura is the largest and most comprehensive study of these issues to date. It has brought to light several issues of physician health that warrant further attention such as nutrition, exercise, sleep and self-care. ${ }^{4}$ The COVID-19 pandemic was an awakening for medical professionals and the ordained reduction in routine work coupled with the inescapable stay at home gave many doctors an opportunity to introspect and focus on their neglected lifestyle.

In our study, $64.3 \%$ respondents reported an improved family life and $46.2 \%$ reported an improvement in eating habits. This could be attributed to less erratic times of eating and consuming more home cooked food during the lockdown period. Similarly, in an Italian survey conducted by Laura Di Renzo et al. on-eating habits and lifestyle changes during the lockdown period, they found a higher adherence to Mediterranean diet, $38.3 \%$ of respondents reported increase in physical activity and $3.3 \%$ quit smoking. ${ }^{5}$

Sleep quality and duration are core to well-being and another aspect that is very often compromised by medical professionals. In our study, $31.4 \%$ of respondents reported an increase in sleep duration versus $24.6 \%$ who reported a decrease in the duration of sleep. This could be due to the lockdown and having to spend a larger part of the day at home along with curtailing of non-emergency work. However, when inquired into the quality of sleep, $31.8 \%$ respondents reported a deterioration in sleep quality while $29.9 \%$ reported an improvement in sleep quality. This may reflect on the psychological impact of the pandemic amongst the participants. In a study by Sara Marelli et al. on the impact of the COVID-19 pandemic on the sleep quality of university students and administrative staff, they found a $24 \%$ prevalence of insomnia, which 
increased to $40 \%$ during the pandemic. ${ }^{6}$ The improvement in sleep quality experienced by the respondents in our study could be attributed to more family time and lesser erratic hours of work.

Regarding physical exercise and fitness in our study with $47 \%$ of respondents reporting better physical fitness during the pandemic. This could be the result of more time at home, having to do all the household chores in the absence of household help and the realisation about the need to improve immunity as self defence against COVID-19. In addition, exercise does improve the sense of well-being and helps mitigate negative emotions and depression. Besides, doctors following a healthy lifestyle are more likely to positively counsel their patients on lifestyle changes (Ref Anna Belgrade). ${ }^{\text {? }}$

\section{Impact on mental health}

Several studies have addressed the issue of mental health in health care professionals. ${ }^{8-13}$ Factors such as the need to educate themselves about the novel virus pandemic, protect themselves and their families, struggle with the discomfort of working in PPEs at work, adapt to change in working schedules and timings, loss of revenue due to curtailment of regular work, etc, have collectively pressurised medical professionals during the pandemic. Our study revealed that at least a quarter of the doctors experienced negative emotions most of the time. Concordantly, Jiangvu Que et al. demonstrated that psychological problems were pervasive amongst the health care workers during the pandemic and the mental health of workers must be protected by timely interventions and proper information feedback..$^{14}$ In another German study which reviewed 14 studies on mental health of health care workers during the pandemic, severe degree of symptoms was found in $2.2 \%$ to $14.5 \%$ of health care workers requiring intervention. ${ }^{15}$ Hence the importance of recognition and seeking intervention for psychological issues in medical professionals during a stressful period like a pandemic cannot be overemphasised.

\section{Impact on livelihood and professional life.}

A substantial proportion of respondents (78.03\%) reported a reduction in monthly income during the pandemic. This could be attributed to the curtailment of all elective procedures and surgeries and decline in patients seeking medical attention for non-emergent conditions. Besides, several doctors with private hospitals had to cease operations due to the impact of travel restrictions on their staff during the lockdown. Investment in procuring protective and sanitising equipment for staff and patient safety further added to the list of expenditures for private clinics. Overall, most doctors bore the brunt of the pandemic on their finances.

The pandemic offered a new window of opportunity in the field of medical education and many webinar based academic meets were conducted. It gave the participants the luxury of attending meetings with renowned faculty across the globe sitting in the comfort of their homes. Almost half of the respondents felt that this form of online learning holds promises for the future as well.

Telephonic consultation which was earlier not approved as a legal means of consultation in the country was approved by the Medical Council of India (MCI) on 25 March 2020 in the wake of the COVID-19 pandemic and guidelines for the same were released for registered medical practitioners. ${ }^{16}$ This brought about a huge change in the consultation practices and patients could now have access to their doctors without risking themselves by travelling and exposing themselves to other patients in hospitals. While most respondents in our study agreed that teleconsultation could not replace a face to face interaction with the patient, $58.5 \%$ of respondents did feel that certain consultations can be conducted online to the benefit of both the patient and the physician even after the pandemic. However, we will need to await the guidelines for the same post pandemic.

The media has reported multiple episodes of discriminatory events against doctors in the form injustices in housing societies and violence from angry relatives of patients in hospitals. However only $8 \%$ of the respondents in our study felt persecuted on account of being a medical professional.

\section{CONCLUSION}

The COVID-19 pandemic has created a tumultuous wave in the personal and professional lives of every human on the planet. While healthcare professionals face a unique set of obstacles and dilemmas that require special attention, many have also experienced a silver lining amidst this chaos in the form of family life and lifestyle changes. Regardless, COVID-19 has been a game changer for everyone!

\section{CONFLICT OF INTEREST}

The authors declare that there is no conflict of interest.

\section{ABBREVIATIONS}

SARS-CoV-2: Severe acute respiratory syndrome virus 2.

\section{REFERENCES}

1. Wikipedia contributors. Covid -19 pandemic in India. In Wikipedia, the Free Encyclopaedia. 2020. Available from: https://en.wikipedia.org/w/index. php?title=COVID-19_pandemic_in_India\&oldid=981737984Jones N, Carver C. Are interventions such as social distancing effective at reducing the risk of asymptomatic healthcare workers transmitting Covid 19 infection to other household members. Nicholas Jones and Catherine Carver on behalf of the Centre for Evidence-based Medicine. 2020. Available from: https://www.cebm. net/covid-19/are-interventions-such-as-social-distancing-effective-at-reducingthe-risk-of-asymptomatic-healthcare-workers-transmitting-covid-19-infectionto-other-household-members/

2. Katie W. Physician Health: A review of lifestyle behaviours and preventive health care amongst physicians. BCMJ. 2012;54(8):419-23.

3. Frank E, Segura C. Health practices of Canadian Physicians. Can Fam Physician. 2009;55(8):810-11

4. Renzo DL, Gualtieri P, Pivari F, Soldati L, Attina A, Cinelli G, et al. Eating habits and lifestyle changes during covid pandemic: An Italian survey. J Transl Med. 2020;18(1):229.

5. Belfrage ASV, Grotmol KS, Tyssen R, Moum T, Finset A, Isaksson K, et al. Factors influencing doctors counselling on patients lifestyle habits: A cohort study. BJGP Open. 2018;2(3):bjgpopen18X101607. Published 2018 Sep 19. Doi:10.3399/bjgpopen18X101607.

6. Marelli S, Catelnuovo A, Somna A, Castronovo V, Mombelli S, Bottoni D, et al. Impact of Covid 19 lockdown on sleep quality in university students and administrative staff. J Neurol. 2020;11:1-8.

7. Yang H, Ma J. How an epidemic outbreak impacts happiness: Factors that worsen (vs protect) emotional wellbeing during the coronavirus pandemic. Psychiatry Res. 2020;89:113045.

8. Ma JF. Impact of Covid 19 pandemic on mental health and quality of life among local residents in Liaoning Province China: A cross sectional study. Int J Environ Res Public Health. 2020;17(7):2381.

9. Zhang Y, Zhang H, MaX, Di Q. Mental health problems during the Covid-19 pandemics and the mitigation effects of exercise: A longitudinal study of college students in China. Int J Environ Res Public Health. 2020;17(10):3722.

10. Balasubramanian A, Paleri V, Benett R, Paleri V. Impact of Covid -19 o the mental health of surgeons and coping strategies. Head Neck. 2020;42(7):1638-44

11. Guo J, Feng $X L$, Wang $X H$, ljzendoorn MV. Coping with Covid 19: Exposure to Covid 19 and negative impact on livelihood predict elevated mental health problems in Chinese adults. Int J Environ Res Public Health. 2020;17(11):3857.

12. Pieh C, Budjmir S, Probst T. The effect of age, gender, income, work and physical activity on mental health during coronavirus disease (Covid 19) lockdown in Austria. J Psychosom Res. 2020;136:110186

13. Que J, Shi L, Deng J, Liu J, Zhang L, Wu S, et al. Psychological impact of the Covid -19 pandemic on healthcare workers: A cross sectional study in China. J Neurosci Ment Health. 2019;19:268-72. 
14. Bohlken J, Schomig F, Lemke M, Pumberger M, Heller S. Covid 19 pandemic: Stress experience of health care workers: A short current review. Psychiatr Prax. 2020;47(4):190-7.

15. Telemedicine Practice Guidelines: Enabling Registered Medical Practioners to
Provide Healthcare Using Telemedicine. Board of Governors, in supersession of the Medical Council; Appendix 5 of the Indian Medical Council (Professional Conduct, Etiquette and Ethics Regulation, 2002). 2020. Available from: https:// www.mohfw.gov.in/pdf/Telemedicine.pdf

Cite this article : Nadkarni T, Patel S, Pawar A, Bodhanwalla M. Impact of COVID-19 Pandemic on the Life of Indian Doctors: Time for Introspection?. Int J Med Public Health. 2021;11(2):85-9. 